\title{
Hormonal and biochemical responses to transcendental meditation
}

\author{
R. Cooper, B.I. Joffe, J.M. Lamprey, A. Botha, R. Shires, S.G. Baker \\ and H.C. Seftel
}

Carbohydrate and Lipid Metabolism Group, Department of Medicine, University of the Witwatersrand Medical School, Johannesburg, South Africa

\begin{abstract}
Summary: This study was designed to assess whether transcendental meditation (TM) could influence various endocrine responses in 10 experienced male meditators. Nine matched subjects, uninformed of the TM procedure, acted as controls. Meditators successfully practised their technique for $\mathbf{4 0} \mathbf{m i n}$ in the morning while controls relaxed for this period. No significant differences emerged between these 2 groups with respect to carbohydrate metabolism (plasma glucose, insulin and pancreatic glucagon concentrations), pituitary hormones (growth hormone and prolactin) or the 'stress' hormones, cortisol and total catecholamines - although meditators tended to have higher mean catecholamine levels. Plasma free fatty acids were significantly elevated in meditators $\mathbf{4 0} \mathrm{min}$ after completing the period of TM. No clear evidence was thus obtained that any of the stress, or stress-related, hormones were suppressed during or after meditation in the particular setting examined.
\end{abstract}

\section{Introduction}

The technique of transcendental meditation (TM) is a simple form of relaxation. It involves sitting with the eyes closed and effortlessly repeating a meaningless sound or 'mantra'. Much of the research available has claimed beneficial effects of TM in a variety of physiological and biochemical measurements (Fenwick, 1983). Of particular interest are reports of reduced levels of urinary catecholamine metabolites and plasma cortisol while practising the technique (Bujatti \& Riederer, 1976; Jevning et al., 1977), although Michaels et al. (1976) found no significant fluctuations in plasma catecholamines during meditation.

In the present investigation we have explored the possible hormonal and biochemical changes during the performance of TM, with special emphasis on the 'stress-related' hormones: catecholamines, cortisol, growth hormone, prolactin, pancreatic glucagon and insulin. These findings were then related to subjective experiences obtained during the meditation period.

R. Cooper, M.B. B.Ch.; B.I. Joffe, F.R.C.P.; J.M. Lamprey; A. Botha; R. Shires, M.R.C.P.; S.G. Baker, M.Sc.; H.C. Seftel, Dip.Med.

Correspondence: B.I. Joffe, Department of Medicine, University of the Witwatersrand Medical School, Parktown, Johannesburg 2193, South Africa.

Accepted: 27 September 1984

\section{Subjects and methods}

Ten non-obese men, aged between 20 and $35 \mathrm{y}$, who were proficient and experienced in the TM technique participated in the study. Nine matched healthy subjects who had never practised TM and who were uninformed of the technique served as controls. None of the meditators nor controls were smokers and the TM group were non-drinkers.

Each individual arrived at the hospital laboratory at 0800 after a $10 \mathrm{~h}$ overnight fast. He was placed in the supine position in a quiet room. A venous cannula was inserted near the antecubital fossa and kept patent with slow-running saline. After $30 \mathrm{~min}$, the subject sat up with his eyes open and remained quiet for $20 \mathrm{~min}$ during which time 2 blood samples were collected - at the beginning and end of this pre-TM period, or prerelaxation period in the controls. Subjects then closed their eyes and the meditators began practising the TM technique, while the controls relaxed. Blood samples were withdrawn after 20 and $40 \mathrm{~min}$ during this $40 \mathrm{~min}$ TM or relaxation phase. Meditators and nonmeditators then opened their eyes and remained quiet for a further $40 \mathrm{~min}$, during which time additional blood samples were taken at 20 and $40 \mathrm{~min}$. Great care was taken to ensure that blood samples were collected in as unobtrusive a manner as possible. The pulse and respiratory rates were recorded at the end of each phase of the study.

(C) The Fellowship of Postgraduate Medicine, 1985 
Table I Pulse and respiratory rates in 10 meditators and 9 non-meditators before, during and after transcendental meditation or relaxation

\begin{tabular}{|c|c|c|c|c|c|c|}
\hline & \multicolumn{3}{|c|}{ Meditators } & \multicolumn{3}{|c|}{ Non-Meditators } \\
\hline & Pre & During & Post & Pre & During & Post \\
\hline $\begin{array}{l}\text { Pulse rate } \\
\text { Respiratory rate }\end{array}$ & $\begin{array}{l}58 \pm 2 \\
12 \pm 1\end{array}$ & $\begin{array}{l}59 \pm 2 \\
12 \pm 1\end{array}$ & $\begin{array}{l}56 \pm 3^{*} \\
11 \pm 1^{* *}\end{array}$ & $\begin{array}{l}67 \pm 4 \\
14 \pm 1\end{array}$ & $\begin{array}{l}65 \pm 5 \\
15 \pm 2\end{array}$ & $\begin{array}{l}67 \pm 4 \\
16 \pm 2\end{array}$ \\
\hline
\end{tabular}

$* P<0.05$ compared with non-meditators

** $P<0.025$ compared with non-meditators

Blood samples were collected into iced tubes, immediately centrifuged and the supernatant plasma deep-frozen until analysis some weeks later. Plasma glucose was estimated using a hexokinase method and free fatty acids by microtitration (Dole, 1956). Plasma total catecholamines (adrenaline and noradrenaline) were determined by a radioenzymatic technique (Passon \& Peuler, 1973). The remaining hormones were measured by radioimmunoassay procedures. These included insulin (Welborn \& Fraser, 1965), pancreatic glucagon, human growth hormone and prolactin (all with commercial kits supplied by Serona Diagnostics, Switzerland); and cortisol, assayed using reagents provided by Amersham International, UK. All hormonal measurements were performed in duplicate in single assay runs.
Subjective experiences were individually recorded after completion of the procedure, according to a standard questionnaire.

Paired and unpaired t-tests, as applicable, were used for the statistical analysis of data. Results are expressed as means \pm s.e.m. in the Table and all the Figures.

\section{Results}

\section{Pulse and respiratory rate (Table $\mathrm{I})$}

The mean pre-meditation/relaxation pulse rate was slightly less in the meditators compared with the controls and it remained slower during the subsequent periods of observation, achieving statistical sig
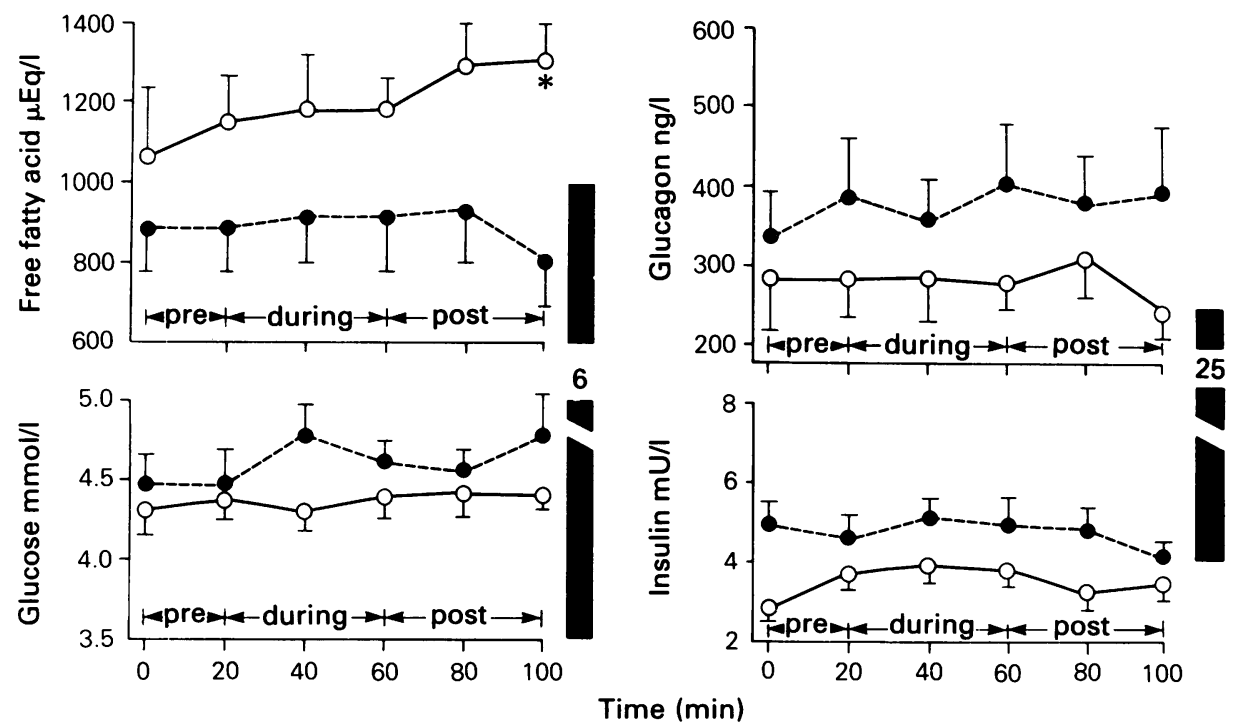

Figure 1 Plasma levels of pancreatic hormones and substrates in 10 meditators $(O)$ and 9 non-meditators (O) before, during and after transcendental meditation or relaxation; $(\boldsymbol{\square})$ normal basal range. Vertical bars indicate standard errors of the mean.

$* P<0.01$ 
nificance by the end of the post-meditation period. A similar trend emerged when the mean respiratory rates were analysed.

\section{Effect on carbohydrate and lipid metabolism (Figure 1)}

Mean plasma glucose concentrations remained fairly constant in both meditators and non-meditators, with no significant difference between the 2 groups at any sampling time. Mean plasma free fatty acids, on the other hand, tended to be higher in the meditating group at all times and this difference reached statistical significance at the end of the post-meditation period $(P<0.01$ compared to the mean control value at that time).

The concentrations of plasma insulin and pancreatic glucagon were similar in both groups throughout the period of observation, mean glucagon levels
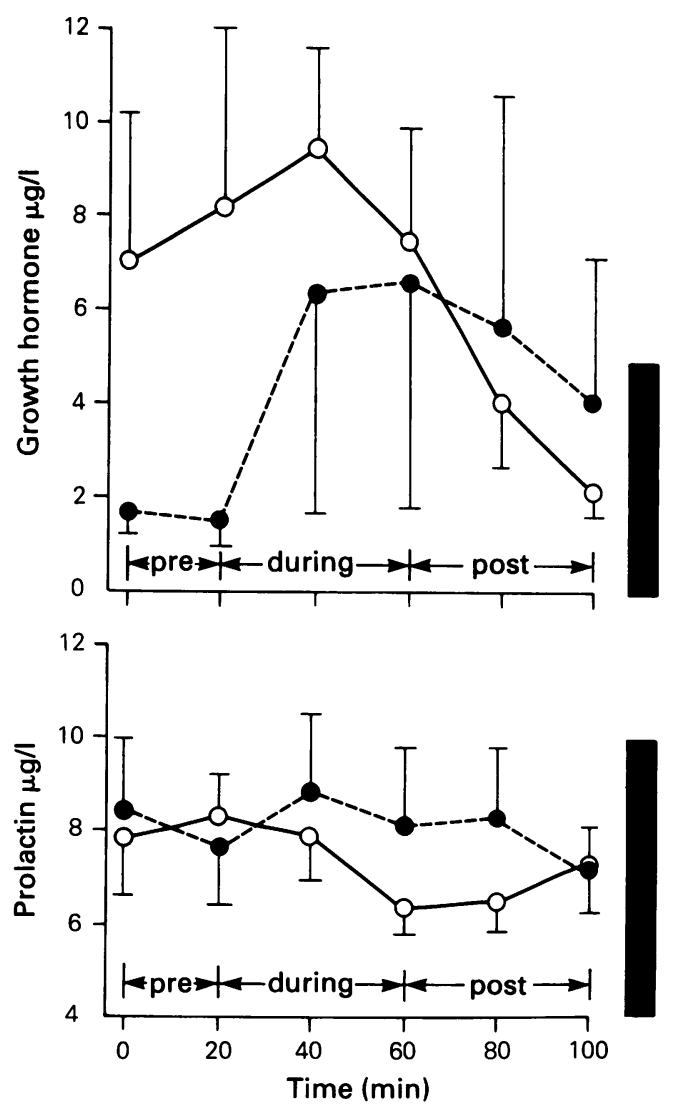

Figure 2 Plasma levels of growth hormone and prolactin in 10 meditators $(O)$ and 9 non-meditators (O) before, during and after transcendental meditation or relaxation; $(\square)$ normal basal range. Vertical bars indicate standard errors of the mean.

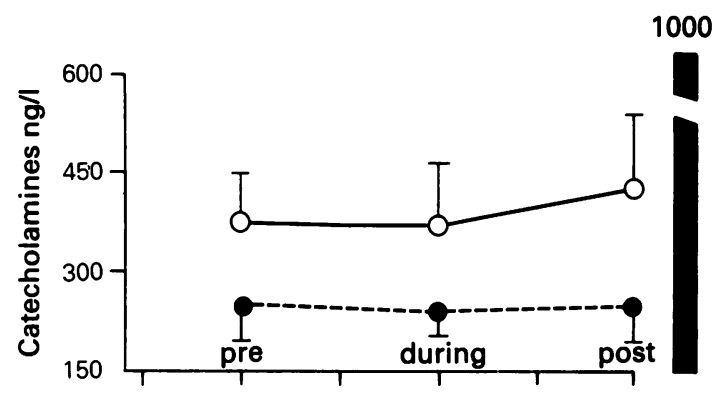

230

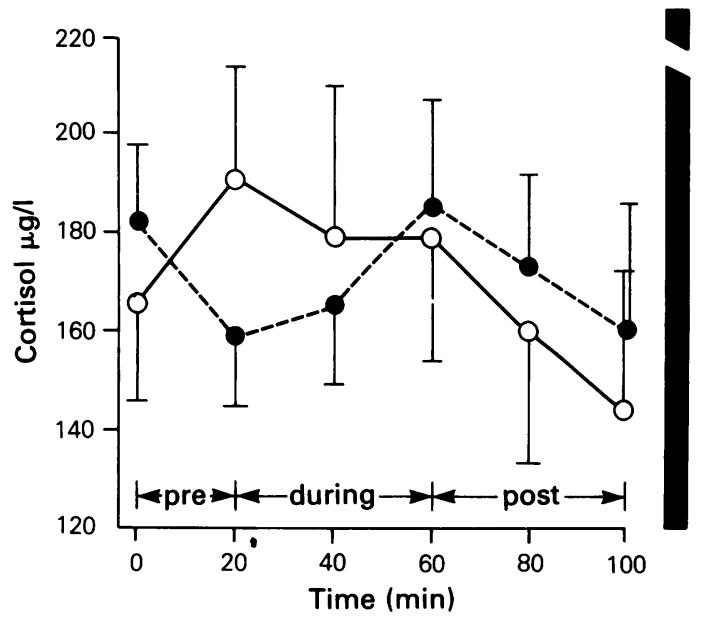

Figure 3 Plasma levels of total catecholamines and cortisol in 10 meditators $(O)$ and 9 non-meditators $(O)$ before, during and after transcendental meditation or relaxation; $(\mathbf{\square})$ normal basal range. Vertical bars indicate standard errors of the mean.

lying slightly above the upper limit of the normal basal range.

\section{Effect on pituitary hormones (Figure 2)}

Growth hormone concentrations fell slightly in both groups during the post-meditation/relaxation period, the trend being more apparent in meditators. However, no significant differences were found at any sampling time either within or between groups. Mean prolactin levels remained unchanged in both groups of subjects.

\section{Effect on 'stress' hormones (Figure 3)}

Plasma catecholamines were measured at only one time during each phase of the study. Their mean concentration was consistently higher in the meditators, but individual results varied widely and statistical significance was not achieved. Mean plasma 
cortisol levels showed no consistent pattern in either group and were not significantly different.

\section{Subjective responses}

All the meditators claimed to have 'transcended' during their period of meditation, and attained the desired sense of mental and physical well-being. They were unaware of their surroundings or any disturbances, such as blood sampling, during meditation. Conversely the non-meditators did not experience any subjective changes during the three stages of the experiment. Some were bored and impatient and kept opening their eyes during the $40 \mathrm{~min}$ relaxation period.

\section{Discussion}

Our study was designed to test whether TM could acutely modify a variety of hormonal and related biochemical responses, as some previous reports have suggested might occur (Bujatti \& Riederer, 1976; Jevning et al., 1977). We found no convincing evidence that any of the stress-related hormones were suppressed in subjects performing the technique at rest. If anything, catecholamine levels were higher in meditators during the study. Furthermore, plasma free fatty acid concentrations, reflecting in part the lipolytic action of catecholamines and other stress hormones (Goodman, 1970), showed a substantial elevation after the period of meditation was completed. This suggests that the state of apparent external relaxation achieved during TM may be accompanied by considerable 'internal' metabolic stimulation or activity.

Could other factors, apart from the TM itself, have influenced the biochemical responses that were

\section{References}

BLACKWELL, B., HANENSON, I., BLOOMFIELD, S., MAGENHEIM, H., GARTSIDE, P., NIDICH, S., ROBINSON, A. \& SIGLER, R. (1976). Transcendental meditation in hypertension. Lancet, i, 223.

BUJATTI, M. \& RIEDERER, P. (1976). Serotonin, noradrenaline, dopamine metabolites in the transcendental meditation technique. Journal of Neural Transmission, 39, 257.

DOLE, V.P. (1956). A relation between non-esterified fatty acids in plasma and the metabolism of glucose. Journal of Clinical Investigation, 35, 150.

FENWICK, P. (1983). Can we still recommend meditation? British Medical Journal, 287, 1401.

GOODMAN, H.M. (1970). Permissive effects of hormones on lipolysis. Endocrinology, 86, 1064.

JEVNING, R., WILSON, A.E., VAN DERLAAN, E. \& LEVINE, S. (1977). Plasma prolactin and cortisol during the transcendental meditation technique. Scientific Research on the Transcendental Meditation Programme: Collected Papers, 1, 143. observed? It is conceivable that the unfamiliar laboratory environment and experimental procedures induced a degree of apprehension in the meditators, with resultant stimulation of the hypothalamopituitary-adrenal axis. Against this criticism are the subjective reports of the meditators claiming successful transcendentation and the objective evidence provided by their slower pulse and respiratory rates. Recording the electroencephalogram (EEG) tracing during meditation might have yielded additional support for this contention, since specific EEG patterns have been described (Wallace, 1970).

Our findings may have clinical relevance. TM has been reported to have therapeutic value in a variety of situations such as relieving psychogenic stress (Throll, 1981), treating insomnia (Woolfolk et al., 1976) and managing patients with systemic hypertension (Blackwell et al., 1976). Whatever benefit may occur in these situations does not appear to be associated with favourable biochemical or hormonal modifications. However, it may be that a reduction in the levels of stress-related hormones might only become apparent when they are already substantially elevated due to environmental stimuli. Moreover, we were unable to measure any of the recently isolated neuro-peptides (such as endorphins) where changes might be anticipated. Nor are we aware of any studies concerning the effect of TM on these peptides.

\section{Acknowledgements}

We thank Dr I. Katzeff and Mrs Marilyn Viljoen for helping with aspects of the study and Mrs $M$. Langridge for preparing the paper for publication. The Department of Medical Biochemistry of the South African Institute for Medical Research kindly measured the plasma glucose levels for us. Financial assistance was provided by the South African Medical Research Council.

MICHAELS, R.R., HUBER, M.J. \& MCCANN, D.S. (1976) Evaluation of transcendental meditation as a method of reducing stress. Science, 192, 1242.

PASSON, P.G. \& PEULER, J.D. (1973). A simplified radiometric assay for plasma norepinephrine and epinephrine. Analytical Biochemistry, 51, 618.

THROLL, D.A. (1981). Transcendental meditation and progressive relaxation: their psychological effects. Journal of Clinical Psychology, 37, 776.

WALLACE, R.K. (1970). Physiological effects of transcendental meditation. Science, 167, 1751.

WELBORN, T.A. \& FRASER, T.R. (1965). The double antibody immunoassay of insulin. Diabetologia, 1, 211.

WOOLFOLK, R., CARR-KAFFESHON, L. \& MCNULTY, T.F. (1976). Meditation training as a treatment for insomnia. Behaviour Therapy, 7, 359. 